\title{
Arquitectura radical y estados fenológicos de cultivos andinos quínoa, amaranto, lupino y alforfón en un Andisol del sur de Chile
}

\author{
Radical architecture and phenological stages of Andean crops quinoa, \\ amaranth, lupine and buckwheat in an Andisol from Southern Chile
}

\author{
Jaime Solano $^{1 *}$, Jorge González, Mauricio Collinao ${ }^{1}$, Fernando Borie $^{1,2}$, Claudia Castillo $^{1,3}$
}

\section{RESUMEN}

En Andisoles del sur de Chile, el rendimiento de los cultivos está principalmente limitado por fijación de fósforo (P) y fitotoxicidad por aluminio $\left(\mathrm{Al}^{3+}\right)$, existieron escasos antecedentes sobre rotaciones con trigo que incluyan cultivos andinos. Resulta relevante el conocimiento de la arquitectura radical en estos cultivos con el fin de mejorar su productividad, al otorgar una mayor capacidad de absorción de agua, nutrientes y tolerancia a estreses bióticos y abióticos. El objetivo del trabajo fue evaluar la arquitectura radical en estadios tempranos de desarrollo de cultivos andinos establecidos en un Andisol del sur de Chile en condiciones de campo. Durante la temporada 2019/2020 se estableció un ensayo en campo utilizando cuatro cultivos: alforfón (Fagopyrum esculentum L.), amaranto (Amaranthus spp.), lupino (Lupinus albus L.) y quínoa (Chenopodium quinoa L.), todos no micorrizables, y como comparación se usó avena (Avena sativa L.), un cultivo micorrizable. En el periodo de crecimiento se realizaron cuatro muestreos de plantas y suelo registrando estadios fenológicos y evaluando arquitectura de raíces, pH del suelo y P disponible. En la primera mitad del ciclo vegetativo, el crecimiento de los cultivos fue continuo y se redujo drásticamente al final del ciclo de desarrollo, por la sequía imperante durante los meses de verano. Estas condiciones climáticas afectaron a quínoa y lupino, los cuales mostraron alta sensibilidad al estrés hídrico. En general, en todos los cultivos se observó una buena arquitectura radical, lo que sugiere una movilización de $\mathrm{P}$ en forma más eficiente. Mayor longitud radical se obtuvo en el alforfón, mientras que mejor capacidad de adaptación al medio se observó en quínoa. Se concluye que, mediante un adecuado manejo agronómico al incorporar quínoa o amaranto como precultivos en rotación con trigo, se podría incrementar la disponibilidad de P para el cultivo siguiente reduciendo la aplicación de fertilizantes químicos.

Palabras clave: cultivos no micorrícicos, WinRhizo, rotación de cultivos, P disponible, longitud total de raíces.

\begin{abstract}
In Andisols of Southern Chile, crop yield is mainly limited by P fixation and aluminum phytotoxicity $\left(A l^{3+}\right)$, but scarce information in these soils is known in rotations, including wheat after Andean crops. It is very relevant to the knowledge of suchcrops' root architecture to improve productivity through a greater absorption capacity of water, nutrients, and tolerance to biotic and abiotic stresses. The objective of this work was to evaluate root architecture at early phenological stages of Andean crops in an acidic Andisol from Southern Chile. During the 2019/2020 season, a field trial was established using four non-mycorrhizal cultures: buckwheat (Fagopyrum esculentum L.), amaranth (Amaranthus spp.), lupine (Lupinus albus L.), and quinoa (Chenopodium quinoa $L$.), and compared with oats (Avena sativa $L$.) a mycorrhizal culture. During the growth period, four plant and soil samplings were carried out, evaluating phenological stages, root architecture, together with soil $p H$ and P-availability. During the first half of the vegetative cycle, the growth of the crops was continuous but drastically reduced at the end development cycle due to the prevailing drought during the summer months. These climatic conditions affected quinoa and lupine growth showing high sensitivity to water stress. In general, all crops showed good root architecture, suggesting a more efficient P mobilization. A greater root length was obtained in buckwheat, while a better adaptation capacity to the environmental conditions was observed in quinoa. It is concluded that through suitable agronomic management by incorporating quinoa or amaranth as pre-crops of wheat in a rotation, the P-availability for the next crop could be increased by reducing the application of chemical fertilizers.
\end{abstract}

Keywords: non-mycorrhizal crops, WinRhizo, crop rotation, available-P, total root length.

\footnotetext{
1 Departamento de Ciencias Agropecuarias y Acuícolas, Facultad de Recursos Naturales, Universidad Católica de Temuco (UC Temuco), Temuco, Chile.

2 Scientific and Technological Bioresource Nucleus, Universidad de La Frontera (UFRO). Temuco, Chile.

3 Núcleo de Investigación en Producción Alimentaria, ,Universidad Católica de Temuco. Temuco, Chile.

* Autor para correspondencia: jsolano@uct.cl
}

Fecha de Recepción: 21 de Octubre, 2020.

Fecha de Aceptación: 17 de Febrero, 2021. 


\section{Introducción}

Entre las principales características que limitan el rendimiento de los cultivos en suelos volcánicos de la macro región sur del país, mayoritariamente Andisoles, se encuentra la elevada actividad de $\mathrm{H}^{+}$ y fitotoxicidad por $\mathrm{Al}^{3+}$, junto con alta fijación de P (Borie et al., 2019). Esto conlleva la aplicación continua de fertilizantes fosfatados para la mantención de una adecuada disponibilidad del nutriente para los cultivos (Luzio, 2010), puesto que su eficiencia de absorción por las plantas no supera al $15 \%$ del $\mathrm{P}$ aplicado un año después de su fertilización (Balemi y Negisho, 2012). $\mathrm{La}$ fitotoxicidad por $\mathrm{Al}^{3+}$ ocasiona alteraciones bioquímicas y fisiológicas en las plantas y, por ende, en su productividad (Mora et al., 2006), causando disminución del crecimiento de las raíces, que se tornan más gruesas y reducen la absorción de agua y nutrientes (Velásquez et al., 2016). Para lograr mayor sostenibilidad en los agroecosistemas es necesario disminuir el uso de fertilizantes químicos con aplicación de nuevas prácticas agrícolas. En el país, los suelos volcánicos soportan la mayor producción de trigo incluyendo habitualmente avena o lupino como precultivo. La avena se utiliza por la relativa tolerancia a $\mathrm{Al}^{3+}$ y el lupino por el aporte de $\mathrm{N}$ y $\mathrm{P}$ mediante exudación de citratos y fosfatasas que hidrolizan $\mathrm{P}$ orgánico (Darch et al., 2016), el cual normalmente es elevado en estos suelos (Borie et al., 2019). Actualmente son escasos los antecedentes sobre los beneficios del uso de cultivos andinos como alforfón (Fagopyrum sculentum Moench.), amaranto (Amaranthus spp.) o quínoa (Chenopodium quinoa Willd.) en rotaciones con trigo. Además, en relación con la productividad de los cultivos, son fundamentales los estudios sobre la geometría radical, ya que una arquitectura adecuada aumenta la capacidad de absorción de agua y nutrientes, proporcionando mayor tolerancia a estreses bióticos y abióticos (Fita et al., 2006). Estudios de arquitectura radical confirman su influencia en la productividad de los cultivos (Lobet et al., 2015) y demuestran la capacidad que tienen las raíces de una determinada especie para explorar un mayor volumen de suelo, estableciendo límites de cantidad de agua o nutrientes potencialmente accesibles a este (Lobet et al., 2015). Esta propiedad variará dependiendo de si la especie vegetal es colonizada por hongos micorrizógenos que forman simbiosis (Smith y Read,
2008). Una hipótesis frecuente es que especies con raíces gruesas, de mayores diámetros, baja densidad radical y cortos pelos radicales obtienen mayor beneficio nutricional producto de la simbiosis al incrementar el volumen de suelo explorado. Sin embargo, algunos antecedentes señalan que no siempre las plantas con poco desarrollo radical tienen una mejor respuesta a la micorrización (Maherali, 2004). Actualmente se reconoce que los hongos micorrizógenos influyen en muchos otros aspectos del crecimiento, estructura y función vegetal, además de la captación de $\mathrm{P}$, agua y otros nutrientes, protección frente a patógenos y herbívoros y resistencia a sustancias tóxicas y estreses ambientales (Begum et al., 2019). Por tanto, el objetivo de este estudio fue evaluar la arquitectura radical de algunos cultivos andinos en estadios tempranos de desarrollo cuando se establecen en condiciones de campo en un Andisol de la región de La Araucanía.

\section{Materiales y métodos}

Durante la temporada agrícola 2019/2020 se estableció un ensayo en un Andisol de la localidad de Pillanlelbún, Temuco, Chile ( $38^{\circ} 39^{\prime} \mathrm{S}$; $\left.72^{\circ} 27^{\prime} \mathrm{O}\right)$ con cuatro cultivos que no forman micorrizas (-MA): a) quínoa var. Regalona-Baer, b) amaranto semilla corriente, c) lupino blanco (Lupinus albus L.) var. Rumbo y d) alforfón cv. Mankán, junto con un cultivo micorrícico (+MA) avena (Avena sativa L.) variedad Supernova, usado como referencia. Las raíces de la avena se asocian con hongos micorrícicos arbusculares (HMA), microorganismos beneficiosos relacionados con la nutrición vegetal, especialmente con mayor absorción de P (Castillo et al., 2012).

Las dosis de semilla $\left(\mathrm{kg} \mathrm{ha}^{-1}\right)$ fueron avena 140, quínoa 12, amaranto 12, lupino 120 y alforfón 30. Las fechas de siembra correspondieron a las recomendadas para cada especie y cultivar en específico, estableciéndose parcelas de $6 \mathrm{~m}^{2}$ con una parcela útil de $1 \mathrm{~m}^{2}$, distribuidas en un diseño de bloques completamente al azar con cuatro repeticiones. La fertilización aplicada a la siembra como N:P:K $\left(\mathrm{kg} \mathrm{ha}^{-1}\right)$ fue para avena 150:78:83, quínoa 50:35:0, amaranto 100:26:17, lupino 0:0:0 y alforfón 40:26:33.

El suelo correspondió a un Andisol $(20 \mathrm{mg}$ $\mathrm{N} \mathrm{kg}^{-1} ; 9$ mg P kg-1; 82 mg K kg-1; $\mathrm{pH} \mathrm{6,05;17 \%}$ MO; $0,21 \mathrm{cmol}_{+} \mathrm{K} \mathrm{kg}^{-1} ; 0,14 \mathrm{cmol}_{+} \mathrm{Na} \mathrm{kg}^{-1} ; 9,72$ 
$\mathrm{cmol}_{+} \mathrm{Ca} \mathrm{kg}^{-1} ; 2,79 \mathrm{cmol}_{+} \mathrm{Mg} \mathrm{kg}-1 ; 0.01 \mathrm{cmol}_{+}$ Al kg-1; 0,06\% saturación Al; CICE de 12,87 $\mathrm{cmol}_{+} \mathrm{kg}^{-1}$, saturación de bases $12,66 \mathrm{cmol}_{+} \mathrm{kg}^{-1}$ ) perteneciente a la serie Temuco (Soil Survey Staff, 1996). El material de origen corresponde a limo sobre grava, sin matriz arenosa, de composición mixta, con una pluviometría que fluctúa entre 1.200 y $2.000 \mathrm{~mm}$. La localidad presenta un drenaje interno y externo medio, con erosión libre y bajo contenido de $\mathrm{P}$, con clima mediterráneo frío templado. En invierno (julio) las temperaturas promedian $4^{\circ} \mathrm{C}$ y en verano (enero) $27^{\circ} \mathrm{C}$. El periodo libre de heladas es de 90 días y comprende los meses de diciembre a febrero. Las precipitaciones medias anuales alcanzan a $1.226 \mathrm{~mm}$, con un déficit hídrico que se extiende entre 3 y 4 meses. Durante el transcurso del ensayo, en el mes de diciembre las temperaturas mínimas fluctuaron entre $-1^{\circ} \mathrm{C}$ y $2^{\circ} \mathrm{C}$, con heladas que dañaron seriamente el cultivo de amaranto.

El ensayo se estableció sobre una pradera natural degradada, realizando la siembra en forma manual y posteriormente, aplicando dos riegos de $20 \mathrm{~mm}$ cada uno, con un sistema de aspersión móvil $\mathrm{K}$-line.

En el cultivo de quínoa, debido a la presencia de mildiú (Peronospora farinosa $\mathrm{f}$. sp. chenopodii Fr.) se realizó una aplicación foliar del fungicida Hortyl 50F (i.a. clorotalonilo) en dosis de $200 \mathrm{~mL}$ $100 \mathrm{~L}^{-1}$ agua y en lupino, por la presencia de Naupactus xanthographus (Curculionidae) se aplicó foliarmente Engeo 247 ZC (i.a. Tiametoxam, Lambda-cihalotrina) en dosis de $200 \mathrm{~mL} \mathrm{ha}^{-1}$.

En la Tabla 1 se observa que se realizaron cuatro muestreos (M1 a M4) para medición de altura desde la base de la planta hasta el extremo apical, evaluando tempranamente arquitectura radical solo en M1 y M2 con mediciones de longitud total de raíces (LTR), área superficial (AS) y número de bifurcaciones mediante el software WinRhizo ${ }^{\circledR}$ (REGENT INSTRUMENT INC, 2003). Para ello, las raíces de las plántulas muestreadas se lavaron cuidadosamente y se colocaron en una bandeja de acrílico transparente con agua distribuyéndolas al azar, para proceder a escanearlas y realizar la lectura con el programa computacional. En el suelo, se determinó P disponible (Kalra y Maynard, 1991) y pH (Sadzawka et al., 2006) en las mismas fechas (M1 a M4) y para este último, se incluyó un quinto muestreo de suelo, posterior a la cosecha (M5).

Para cada cultivo se realizó un seguimiento de los estadios fenológicos (Bleiholder et al., 1998) usando la escala $\mathrm{BBCH}$ expandida. La cosecha de la parcela útil $\left(1 \mathrm{~m}^{2}\right)$ se hizo en madurez fisiológica cuando los cultivos presentaron sobre 95\% de los granos secos, cortando las plantas a una altura de $10 \mathrm{~cm}$ desde el suelo. Las semillas se separaron en forma manual eliminando impurezas mediante tamizado y aplicación de aire en forma superficial. El rendimiento por hectárea, de cada cultivo, se determinó a partir de todas las semillas limpias, separadas de la inflorescencia y/o espiga y pesadas en balanza de precisión.

Los resultados obtenidos se sometieron a una prueba de normalidad y homogeneidad. Luego, se realizó ANDEVA y las diferencias entre medias fueron sometidas a la prueba de comparación múltiple de Tukey $(\mathrm{p} \leq 0,05)$, mediante el software SPSS versión 15.0.

\section{Resultados y discusión}

En la Tabla 2 se muestra la fenología de los cultivos establecidos en agosto (avena, lupino) y octubre (alforfón, amaranto, quínoa) de la temporada agrícola 2019. En avena, el desarrollo vegetativo con formación de macollo alcanzó

Tabla 1. Fechas de siembra, muestreos y cosecha de los cultivos.

\begin{tabular}{|c|c|c|c|c|c|c|c|c|}
\hline \multirow{2}{*}{ Cultivo } & \multirow{2}{*}{ Fecha siembra } & \multirow{2}{*}{$\frac{\text { Período crecimiento }}{\text { (DDS) }}$} & \multicolumn{5}{|c|}{ Muestreo (DDS) } & \multirow{2}{*}{ Fecha cosecha } \\
\hline & & & $\overline{\mathrm{M} 1}$ & M2 & M3 & M4 & $\overline{\text { M5 }}$ & \\
\hline Avena & $12 / 08 / 2019$ & 162 & 85 & 98 & 114 & 150 & 42 & $21 / 01 / 2020$ \\
\hline Quínoa & $3 / 10 / 2019$ & 145 & 33 & 46 & 62 & 98 & 7 & $25 / 02 / 2020$ \\
\hline Amaranto & $3 / 10 / 2019$ & 70 & 33 & 46 & 62 & 98 & 85 & $*$ \\
\hline Lupino & $12 / 08 / 2019$ & 182 & 85 & 98 & 114 & 150 & 22 & $10 / 02 / 2020$ \\
\hline Alforfón & $3 / 10 / 2019$ & 98 & 33 & 46 & 62 & 98 & 54 & $9 / 01 / 2020$ \\
\hline
\end{tabular}

DDS: días después de la siembra; *No se realizó cosecha, plantas dañadas por heladas. 
Tabla 2. Estados fenológicos de los cultivos en función de los días después de la siembra (DDS).

\begin{tabular}{|c|c|c|c|c|c|}
\hline Cultivo & Parámetro & M1 & M2 & M3 & M4 \\
\hline & $\mathrm{BBCH}$ & 31 & 40 & 55 & 89 \\
\hline Avena & $\begin{array}{l}\text { Estado } \\
\text { fenológico }\end{array}$ & $\begin{array}{l}\text { Encañado. } \\
1^{\text {er }} \text { nudo a } 1 \mathrm{~cm} \\
\text { sobre nudo } \\
\text { macollaje. }\end{array}$ & $\begin{array}{l}\text { Hoja bandera } \\
\text { desarrollada. } \\
\text { Lígula recién visible. } \\
\text { Embuchamiento. }\end{array}$ & $\begin{array}{l}\text { Mitad espigado. } \\
\text { Emergencia mitad de } \\
\text { panícula. }\end{array}$ & $\begin{array}{l}\text { Madurez completa. } \\
\text { Grano duro. }\end{array}$ \\
\hline & $\mathrm{BBCH}$ & 18 & 40 & $50-59$ & 85 \\
\hline Quínoa & $\begin{array}{l}\text { Estado } \\
\text { fenológico }\end{array}$ & 8hv desplegadas. & $\begin{array}{l}\text { Desarrollo partes } \\
\text { vegetativas. Elongación } \\
\text { tallos. } 4 \text { brotes laterales. } \\
4 \text { nudos. }\end{array}$ & $\begin{array}{l}\text { Inflorescencia } \\
\text { encerrada por hojas, } \\
\text { flores aún cerradas. } \\
\text { ( }<7 \text { nudos). }\end{array}$ & $\begin{array}{l}\text { Grano grueso, blanco } \\
\text { pastoso. Pericarpio } \\
\text { rojo o negro. }\end{array}$ \\
\hline \multirow[b]{2}{*}{ Amaranto } & $\mathrm{BBCH}$ & 12 & 17 & 30 & 51 \\
\hline & $\begin{array}{l}\text { Estado } \\
\text { fenológico }\end{array}$ & $\begin{array}{l}\text { Desarrollo } \\
\text { foliar, } 2 \mathrm{hv}\end{array}$ & Desarrollo foliar, $7 \mathrm{hv}$ & Desarrollo foliar, $10 \mathrm{hv}$ & $\begin{array}{l}\text { Sobrevivientes. } 16 \mathrm{hv.} \\
1 \text { flor. }\end{array}$ \\
\hline \multirow[b]{2}{*}{ Lupino } & $\mathrm{BBCH}$ & 30 & $32-38$ & 65 & 70 \\
\hline & $\begin{array}{l}\text { Estado } \\
\text { fenológico }\end{array}$ & Plántula. & $\begin{array}{l}\text { Inicio alargamiento tallo. } \\
\text { Desarrollo ejes } \\
\text { principales y laterales. }\end{array}$ & $\begin{array}{l}\text { Floración. } \\
\text { Eje principal 100\% } \\
\text { florecido. } \\
\text { Inicio floración lateral. }\end{array}$ & $\begin{array}{l}\text { Primeras vainas } \\
\text { alcanzan longitud } \\
\text { final. } \\
\text { Fructificación. }\end{array}$ \\
\hline & $\mathrm{BBCH}$ & 14 & 15 & $36-70$ & 97 \\
\hline Alforfón & $\begin{array}{l}\text { Estado } \\
\text { fenológico }\end{array}$ & $\begin{array}{l}\text { 4hv desplegadas } \\
4 \text { nudos. }\end{array}$ & $\begin{array}{l}5 \text { a } 6 \text { hv desplegadas. } \\
5 \text { nudos. }\end{array}$ & $\begin{array}{l}6 \text { hv desplegadas. } 6 \\
\text { nudos. Primeros frutos } \\
\text { verdes: aquenios verdes } \\
\text { visibles. }\end{array}$ & $\begin{array}{l}\text { Planta madura: tallos } \\
\text { se tornan marrones, } \\
\text { secos y frágiles. }\end{array}$ \\
\hline
\end{tabular}

hv: hojas verdaderas.

84 d, con una etapa reproductiva de más de $60 \mathrm{~d}$. Un comportamiento similar se observó en lupino, con un período vegetativo de aproximadamente $95 \mathrm{~d}$ y etapa reproductiva superior a $80 \mathrm{~d}$. En el caso de quínoa, la etapa vegetativa duró $46 \mathrm{~d}$ y la reproductiva abarcó un periodo superior a 90 d, hasta senescencia. En cambio, amaranto presentó una extensa etapa vegetativa, de $100 \mathrm{~d}$, y fue afectado por las heladas, con fuerte necrosis foliar y pérdida de follaje, con plantas sobrevivientes que alcanzaron la floración, a los $100 \mathrm{~d}$.

Los estadios principales de crecimiento no siempre ocurren en secuencia. También puede darse el desarrollo de varias fases al mismo tiempo. En el país no existen estudios en campo sobre la fenología de alforfón, amaranto o quínoa. Solo se tienen antecedentes de la fenología de quínoa con dos genotipos chilenos: Ancovinto y Chiloé, cultivados en invernadero, con condiciones controladas de temperatura, luz y humedad (Sosa-Zúñiga et al., 2017).
El alforfón es un cultivo muy precoz, con un rango de 49 a $70 \mathrm{~d}$, desde la siembra a la madurez. Se desarrolla en una amplia variedad de suelos y se adapta a condiciones nutricionalmente limitantes (Parodi y Nebreda, 1998). En este estudio el cultivo de alforfón tuvo un desarrollo vegetativo que abarcó 60 d, observándose una abundante biomasa vegetal, mientras que el inicio de la etapa reproductiva no fue uniforme, semejante a la maduración que fluctuó entre 60 y $90 \mathrm{~d}$ (Cuadro 2). Zhou et al. (2018) reportaron valores promedio de 60 a $77 \mathrm{~d}$ para el crecimiento vegetativo con altura de planta de $107 \mathrm{~cm}$. También en alforfón, Rauf et al. (2020), en una evaluación realizada con 250 accesiones pertenecientes a una colección de germoplasma basada en caracteres agromorfológicos, señalaron que para alcanzar $50 \%$ de floración y $80 \%$ de madurez, los tiempos variaron entre 38 y $45 \mathrm{~d}$ y de 73 a 95 d, respectivamente. Además, identificaron accesiones promisorias de temprana maduración, 
con un ciclo inferior a $80 \mathrm{~d}$. La fenología observada en el cultivo de alforfón permite proyectar que en rotación con trigo, podría incrementar la rentabilidad al utilizar menores recursos generando estrategias competitivas.

En la Tabla 3, al observar la arquitectura radical, en M2, LTR siguió la secuencia avena>alforfón> quínoa>lupino>amaranto, mientras que para el AS fue avena>lupino $>$ alforfón $>$ quínoa $>$ amaranto. La LTR es uno de los principales parámetros para estimar la cantidad de nutrientes y agua extraídos por la planta en un período determinado. La mayor AS de las raíces la presentó avena (cultivo de referencia), seguida por lupino y alforfón, probablemente debido a las características pivotantes que tienen las raíces de estos dos cultivos (-MA), mientras que aquellos pertenecientes a la familia Amaranthaceae (quínoa y amaranto) mostraron la menor AS. El más alto número de bifurcaciones se obtuvo en las raíces de avena y el más bajo en amaranto. El cultivo con mejor arquitectura radical fue la avena (+MA), cereal usado como referencia, que a semejanza del trigo se adapta a suelos ácidos presentando una cierta tolerancia a la toxicidad por Al y a la vez desarrolla raíces primarias más profundizadoras que aumentan la longitud de la raíz, permitiéndole enfrentar de mejor forma el estrés por sequía.
En la primera mitad del ciclo vegetativo (M1M2), las plantas de todos los cultivos tuvieron un crecimiento continuo (Tabla 3). No obstante, hacia el final del ciclo de desarrollo (M4), la altura de planta se redujo fuertemente a causa de la sequía que se registró en los meses de verano (diciembre a febrero) (datos no presentados). Estas condiciones climáticas afectaron principalmente a la quínoa y lupino, cultivos que mostraron una alta sensibilidad a la escasez de agua.

En todos los cultivos los rendimientos fueron bajos, debido probablemente a las intensas sequías imperantes durante los últimos 10 años en el país y que se acrecentaron en el último año, presentando la temporada 2019 un déficit hídrico superior al $50 \%$ respecto a un año normal (Tabla 4).

El rendimiento del cultivo de quínoa estuvo dentro de los rangos informados por Garrido et al. (2013), quienes reportaron en diferentes genotipos de quínoa rendimientos entre 750 y $2.030 \mathrm{~kg} \mathrm{ha}^{-1} \mathrm{y}$ menor a los observados en otras localidades cercanas como Vilcún y Tranapuente con rendimientos superiores a los 3.000 y $3.800 \mathrm{~kg} \mathrm{ha}^{-1}$ (Mera et al., 2018). Por otro lado, la quínoa es susceptible a heladas tardías de primavera y tempranas de otoño, deteniendo el crecimiento con temperaturas inferiores a $10^{\circ} \mathrm{Co}$ mayores a $20^{\circ} \mathrm{C}$, manteniendo

Tabla 3. Altura de planta y arquitectura radical de los cultivos en campo.

\begin{tabular}{lrrrrrrrr}
\hline \multirow{2}{*}{ Cultivo } & \multicolumn{2}{c}{ Altura $(\mathrm{cm})$} & \multicolumn{2}{c}{ LTR $(\mathrm{cm})$} & \multicolumn{2}{c}{ AS $(\mathrm{cm})$} & \multicolumn{2}{c}{ N $^{\circ}$ bifurcaciones } \\
\cline { 2 - 8 } & \multicolumn{1}{c}{ M1 } & M2 & \multicolumn{1}{c}{ M1 } & M2 & M1 & M2 & M1 & M2 \\
\hline Avena & 43,0 & 73,0 & 480 & 600 & 80 & 105 & 2500 & 3800 \\
Quínoa & 8,0 & 22,8 & 90 & 280 & 13 & 40 & 400 & 1700 \\
Amaranto & 5,0 & 17,8 & 30 & 110 & 10 & 15 & 350 & 600 \\
Lupino & 20,0 & 42,3 & 170 & 190 & 70 & 75 & 750 & 1500 \\
Alforfón & 15,0 & 43,0 & 200 & 350 & 20 & 70 & 1000 & 2400 \\
\hline
\end{tabular}

M1, M2: muestreos; LTR: longitud total de raíces; AS: área superficial.

Tabla 4. Rendimiento de los cultivos y población de plantas, a la cosecha en campo.

\begin{tabular}{lcc}
\hline Cultivo & Población de plantas $\left(\right.$ plantas $\left.\mathrm{m}^{-2}\right)$ & Rendimiento $\left(\mathrm{kg} \mathrm{ha}^{-1}\right)$ \\
\hline Avena & 473 & 4.250 \\
Quínoa & 57 & 1.874 \\
Amaranto & 46 & $\mathrm{ND}$ \\
Lupino & 33 & 1.513 \\
Alforfón & 44 & 1.023 \\
\hline
\end{tabular}

ND: Cultivo dañado por heladas. 
constante la tasa de crecimiento. Según Barriga et al. (1988), siembras muy tempranas del cultivo podrían sufrir riesgo de heladas, por lo que en las zonas centro-sur y sur del país se debe sembrar a fines de noviembre como cultivo de primavera, ya que la precocidad le permite madurar antes de las primeras heladas de otoño. En relación con el cultivo de alforfón, el rendimiento obtenido en el ensayo estuvo dentro de los rangos alcanzados internacionalmente y que fluctúan entre 500 y $4.000 \mathrm{~kg} \mathrm{ha}^{-1}$ (Espig, 1990).

En la Tabla 5 se observa el pH del suelo determinado en cada muestreo para cada uno de los cultivos, encontrándose diferencias en todas las fechas evaluadas. La menor acidez la mostró el alforfón durante la cosecha, pero sin diferencias significativas con amaranto. En general, en todos los cultivos la mayor acidez del suelo se observó al inicio del ciclo vegetativo, lo que podría asociarse a la fertilización amoniacal aplicada en la siembra, mientras la avena fue el que presentó menor acidez al comienzo del ciclo (M1 y M2). Hay que señalar que la fertilización acidificó el suelo original $(\mathrm{pH} 6,05)$ y a la cosecha, la acidez de los cultivos en orden creciente correspondió a la secuencia alforfón $>$ amaranto $>$ lupino $>$ quínoa. Los valores de $\mathrm{pH}$ más altos se observaron entre M1 y M3 con el cultivo de lupino, probablemente porque no recibió fertilización base y por la posible exudación de ácidos quelantes por parte de sus raíces. Se esperaba una mayor acidificación producida por las raíces proteoídeas del cultivo $\mathrm{y}$, por tanto, un mayor efecto en el $\mathrm{P}$ disponible, lo que pudiera deberse a una característica de la variedad de lupino utilizada, lo cual convendría profundizar. Durante las fases de crecimiento, los efectos modificadores del $\mathrm{pH}$ en el suelo fueron menores en los cultivos de ciclo vegetativo corto junto con lupino, que lo acidificó significativamente durante la floración.

En el contenido de P disponible del suelo se observa que en todos los cultivos se encontraron diferencias significativas, a excepción de avena (Tabla 5). En general, en los cultivos entre fructificación y senectud hubo un incremento importante de $\mathrm{P}$ en el suelo, especialmente para la quínoa. Esto podría explicarse por las diferentes dosis de fertilización fosfatada que recibió cada cultivo en el momento de la siembra. La mayor fertilización fosfatada se aplicó a la avena con $78 \mathrm{~kg} \mathrm{ha}^{-1} \mathrm{y}$ fue el cultivo que presentó mayor nivel de $\mathrm{P}$, en marzo del año siguiente $13,21 \mathrm{mg} \mathrm{kg}^{-1}$. Los niveles de $\mathrm{P}$ disponible en el suelo proveniente de quínoa, amaranto y alforfón fueron 9,7; 9,5 y 7,9 $\mathrm{mg} \mathrm{kg}^{-1}$, respectivamente. Si bien estos valores son inferiores a los de avena, las dosis de fertilización aplicadas a cada cultivo fueron menores y se redujeron en más del $50 \%$, correspondiendo a 35,26 y $26 \mathrm{~kg} \mathrm{ha}^{-1}$ para quínoa, amaranto y alforfón. Un aspecto importante es que en quínoa y amaranto los niveles finales de $\mathrm{P}$ fueron superiores en 0,7 y 0,5 $\mathrm{mg} \mathrm{kg}-1$, en comparación con el valor inicial del suelo $\left(9 \mathrm{mg} \mathrm{kg}^{-1}\right)$ mientras que, el lupino mantuvo durante todo su ciclo vegetativo el nivel original. A la cosecha, el lupino movilizó todo el nutriente a la planta, permaneciendo en el suelo solo $3,2 \mathrm{mg}$ $\mathrm{P} \mathrm{kg}^{-1}$, lo que podría afectar la disponibilidad del nutriente para el cultivo siguiente de la rotación.

Tabla 5. pH y P disponible en el suelo de los cultivos en campo.

\begin{tabular}{|c|c|c|c|c|c|c|}
\hline Parámetro & Cultivo & M1 & M2 & M3 & M4 & M5 \\
\hline \multirow{5}{*}{$\mathrm{pH}$} & Avena & $5,44 \mathrm{ab} * \mathrm{AB} * *$ & $5,64 \mathrm{a} A$ & $5,26 \mathrm{~cd} \mathrm{~B}$ & $5,26 \mathrm{a} B$ & $5,32 \mathrm{~b} \mathrm{~B}$ \\
\hline & Quínoa & $4,86 \mathrm{c} \mathrm{D}$ & $5,14 \mathrm{~b} \mathrm{C}$ & $5,58 \mathrm{~b} \mathrm{~A}$ & $5,07 \mathrm{~b} \mathrm{C}$ & $5,41 \mathrm{ab} \mathrm{B}$ \\
\hline & Amaranto & $4,97 \mathrm{c} \mathrm{C}$ & $5,01 \mathrm{bc} \mathrm{C}$ & $5,35 \mathrm{c} \mathrm{B}$ & $4,69 \mathrm{c} \mathrm{D}$ & $5,67 \mathrm{ab} \mathrm{A}$ \\
\hline & Lupino & $5,72 \mathrm{a} \mathrm{B}$ & $5,67 \mathrm{a} \mathrm{B}$ & $6,05 \mathrm{a} A$ & $5,24 \mathrm{a} \mathrm{C}$ & 5,56ab B \\
\hline & Alforfón & $5,18 \mathrm{bc} \mathrm{B}$ & $5,58 \mathrm{a} \mathrm{AB}$ & $5,53 \mathrm{~b} \mathrm{AB}$ & $5,05 \mathrm{~b} \mathrm{~B}$ & $5,94 \mathrm{a} \mathrm{A}$ \\
\hline \multirow{5}{*}{$\mathrm{P}$ disponible $\left(\mathrm{mg} \mathrm{kg}^{-1}\right)$} & Avena & $18,60 \mathrm{~b} \mathrm{~A} *$ & $17,60 \mathrm{a} \mathrm{A}$ & $24,05 \mathrm{a} A$ & $13,21 \mathrm{a} \mathrm{A}$ & \\
\hline & Quínoa & $40,86 \mathrm{a} A$ & $12,62 \mathrm{bc} \mathrm{B}$ & $18,31 \mathrm{ab} B$ & 9,69ab B & \\
\hline & Amaranto & $20,40 \mathrm{~b} \mathrm{~A}$ & $12,17 \mathrm{bc} A B$ & $13,29 \mathrm{~b} \mathrm{AB}$ & $9,54 \mathrm{ab} \mathrm{B}$ & \\
\hline & Lupino & $9,68 \mathrm{~b} \mathrm{~B}$ & $8,28 \mathrm{c} \mathrm{B}$ & $13,88 \mathrm{~b} \mathrm{~A}$ & $3,17 \mathrm{c} \mathrm{C}$ & \\
\hline & Alforfón & $14,00 \mathrm{~b} \mathrm{~A}$ & $11,62 \mathrm{c} \mathrm{AB}$ & $13,40 \mathrm{~b} \mathrm{~A}$ & $7,88 \mathrm{~b} \mathrm{~B}$ & \\
\hline
\end{tabular}

M1, M2, M3, M4; M5: muestreos.

*Letras minúsculas distintas en columnas señalan diferencias significativas según test de Tukey $(\mathrm{p} \leq 0,05)$ entre cultivos.

**Letras mayúsculas en filas distintas señalan diferencias significativas según test de Tukey $(\mathrm{p} \leq 0,05)$ entre muestreos. 
Por el contrario, la disponibilidad de $\mathrm{P}$ en el suelo en quínoa y amaranto no presentó diferencias significativas con la avena (M4), probablemente debido a una mayor solubilización y movilización del nutriente, lo que hace necesario estudiar más en detalle los posibles mecanismos involucrados (Tabla 5). Al no observarse diferencias significativas en el pH rizosférico en estos cultivos (M5), pudieran estar actuando las fosfatasas, enzimas que mineralizarían $\mathrm{P}$ orgánico, el cual es alto en este tipo de suelos (Borie et al., 2019). De allí que, en rotaciones de cultivo con trigo, se podría reemplazar el precultivo de avena por quínoa o amaranto, para elevar la disponibilidad de P y, por tanto, reducir la fertilización fosfatada. Otro aspecto interesante es que en el suelo después de la cosecha, los mayores niveles de $\mathrm{P}$ los presentaron los cultivos de ciclo corto (amaranto, quínoa, alforfón), excepto avena (ciclo largo), que fueron sembrados a comienzos de octubre de 2019.

En general, de acuerdo a los resultados obtenidos en los cinco cultivos, se concluye que una mejor arquitectura radical permitiría una movilización más eficiente del P. Por otro lado, se confirmó la capacidad de adaptación de la quínoa a diversos tipos de estreses como bajas temperaturas, sequía y niveles deficitarios de nutrientes. En el caso de lupino, los resultados fueron contrarios a los informados en la literatura, que señalan que junto con la capacidad de crecimiento desarrollada en suelos sometidos a tensiones ambientales, esta especie solubiliza/hidroliza gran cantidad de $\mathrm{P}$ en suelos con baja disponibilidad (Dissayanaka et al., 2017). En el caso de alforfón, se reportan propiedades nutricionales sobresalientes y un rol importante como solubilizador de P (MartínezVillaluenga et al., 2020), efecto que tal vez se hubiera observado con una aplicación menor de $\mathrm{P}$ fertilizante.

\section{Conclusiones}

Los cinco cultivos mostraron un buen comportamiento agronómico, a pesar de que por efecto de la sequía los rendimientos fueron bajos. Los cultivos andinos alcanzaron la madurez fisiológica en un ciclo de 100 a 120 días, a excepción del amaranto que fue dañado por las heladas. El alforfón, con un desarrollo vegetativo de 60 días, presentó abundante biomasa, manteniéndose la etapa reproductiva hasta los 90 días y mostrando un ciclo corto de rápido crecimiento, con adaptación al Andisol.

Los distintos cultivos presentaron una arquitectura radical que permitió movilizar más eficientemente el $\mathrm{P}$ desde el suelo. La mayor longitud total de raíces se observó en alforfón, mientras que el menor número de bifurcaciones y reducida longitud radical correspondió a amaranto. Mediante un manejo agronómico adecuado de rotaciones incluyendo quínoa o amaranto como precultivos en una rotación, podría verse aumentada la disponibilidad de $\mathrm{P}$ para el cultivo siguiente, reduciendo la aplicación de fertilizantes químicos.

\section{Agradecimientos}

Se agradece al proyecto FONDECYT No1191551 "How precedent non-mycorrhizal crops affect soil $\mathrm{P}$ bioavailability, physiological root traits, and mycorrhizal symbiosis of wheat in a rotation system in volcanic soils of Chile", ANID, Chile, por el financiamiento otorgado para la ejecución del estudio.

\section{Literatura citada}

Balemi, T.; Negisho, K.

2012. Management of soil phosphorus and plant adaptation mechanisms to phosphorus stress for sustainable crop production: A review. Journal Soil Science and Plant Nutrition, 12: 547-562.

Barriga, P.; Fuentes, R.; Segovia, J.; Thomberty, J.; Manríquez, M. 1988. Rendimiento y calidad del alforfón (Fagopyrum esculentum Moench.) en el sur de Chile. Agro Sur, 16: 94-102.

Begum, N.; Cheng, K.; Abass, A.M.; Raza, S.; Ishfaq, KM.; Ashraf, M.; Ahmed, N.; Zhang, L.

2019. Role of arbuscular mycorrhizal fungi in plant growth regulation: Implications in abiotic stress tolerance. Frontiers Plant Science. 10:1068. doi: 10.3389/fpls.2019.01068.
Bleiholder, H.; Meier, V.; van den Boom, T.; Buhr, L.; Hack, H.; Klose, R.; Stauss, R.; Weber, E.

1998. Compendio para la identificación de los estadios fenológicos de especies mono- y dicotiledóneas cultivadas. Escala BBCH extendida. BBA BSA IGZ IVA AgrEvo BASF Bayer Novartis. Limburgerhof, Alemania. 123 p.

Borie, F.; Aguilera, P.; Castillo, C.; Valentine, A.; Seguel, A.; Barea, J.M.; Cornejo, P.

2019. Revisiting the nature of phosphorus pools in Chilean volcanic soils as a basis for arbuscular mycorrhizal management in plant P acquisition. Journal of Soil Science and Plant Nutrition, 19: 390-401. 
Castillo, C.G.; Puccio, F.; Morales, D.; Borie, F.; Sieverding, E. 2012. Early arbuscular mycorrhizal colonization of wheat, barley and oats in Andosols of Southern Chile. Journal Soil Science and Plant Nutrition, 12: 511-524.

Darch, T.; Blackwell, M.S.A.; Chadwick, D.; Hawkins, J.M.B.;

Turner, B.L.

2016. Assessment of bioavailable organic phosphorus in tropical forest soils by organic acid extraction and phosphatase hydrolysis. Geoderma, 284: 93-102.

Dissayanaka, S.; Wickramasinghe, W.M.K.R.; Marambe, D.; Wasaki, J.

2017. Phosphorus-mobilization strategy based on carboxylate exudation in lupin (Lupinus, Fabaceae): A mechanism facility the growth and phosphate acquisition of neighboring plants under phosphate-limited conditions. Experimental Agriculture, 53(2): 308-319.

Espig, G.

1990. A plea for pseudocereals buckwheat, quinoa, and amaranthus. Field Crops, 43: 1.

Fita, A.; Picó, B.; Nuez, F.

2006. Implications of the genetics of root structure in melon breeding. Journal of American Society Horticultural Science, 131: 372-379.

Garrido, M.; Silva, P.; Silva, H.; Muñoz, R.; Baginsky, C.; Acevedo, E.

2013. Evaluación del rendimiento de nueve genotipos de quinua (Chenopodium quinoa Willd.) bajo diferentes disponibilidades hídricas en ambiente mediterráneo. IDESIA, 31(2): 69-76.

Kalra, Y.P.; Maynard, D.G.

1991. Methods manual for forest soil and plant analysis. Micromedia Ltda. Canadá. 116 p.

Lobet, G.; Pound, M.P.; Diener, J.; Pradal, C.; Draye, X.; Godin,

C.; Javaux, M.; Leitner, D.; Meunier, F.; Nacry, P.; Pridmore,

T.P.; Schnepf, A.

2015. Root system markup language: Toward a unified root architecture description language. Plant Physiology, 167: 617-627.

Luzio, W.

2010. Suelos de Chile. Maval Impresores. Santiago, Chile. 364 p.

Maherali, $\mathrm{H}$.

2004. Is there an association between root architecture and mycorrhizal growth response? New Phytologist, 204: 192-200.
Martínez-Villaluenga, C.E.; Peñas, E.; Hernández-Ledesma, B. 2020. Pseudocereal grains: Nutritional value, health benefits and current applications for the development of gluten-free foods. Food and Chemical Toxicology, 137: 111178. doi: 10.1016/j.fct.2020.111178.

Mera, M.; Alcalde, J.M; von Baer, I.

2018. Establecimiento de quínoa (Chenopodium quinoa Willd.). Informativo 95. Instituto de Investigaciones Agropecuarias, INIA Carillanca. Temuco, Chile. 2 p.

Mora, M.; Jarvis, S.; Cartes, P.

2006. Soil Al availability in Andisols of Southern Chile and its effects in forage production and animal metabolism. Soil Use Management, 22: 95-101.

Parodi, P.; Nebreda, I.

1998. Alforfón (Fagopyrum esculentum Moench.) valor nutritivo, usos, fitosanidad y manejo agronómico. Ciencia e Investigación Agraria, 25(2): 91-101.

Rauf, M.; Yoon, H.; Lee, S.; Hyun, D.; Lee, M.; Oh, S.; Choi, Y. 2020. Evaluation of Fagopyrum esculentum Moench. germplasm based on agro-morphological traits and the rutin and quercetin content of seeds under spring cultivation. Genetic Resources and Crop Evolution, 67: 1385-1403.

Sadzawka, A.; Carrasco, M.; Grez, R.; Mora, M.L.

2006. Métodos de análisis de tejidos vegetales. Comisión de Normalización y Acreditación. Sociedad Chilena de la Ciencia del Suelo. INIA La Platina. Santiago, Chile. 53 p.

Smith, S.; Read, D.

2008. Mycorrhizal symbiosis. $3^{\text {rd }}$ edition. Academic Press. New York, EE.UU. 800 p.

Soil Survey Staff.

1996. Soil survey laboratory methods manual. Soil Survey Investigations Report, vol. 42. USDA SCS. Washington DC, USA.

Sosa-Zúñiga, V.; Brito, V.; Fuentes, F.; Steinfort, U.

2017. Phenological growth stages of quinoa (Chenopodium quinoa) based on the BBCH scale. Annals of Applied Biology, 171(1): 117-124.

Velásquez, G.; Rumpel, C.; Redel, J.; Condron, L.M.; Thi Ngo,

P.; Calabi-Floody, M.; Turner, B.; Mora, M.L.

2016. Chemical nature of residual phosphorus in Andisols. Geoderma, 271: 27-31.

Zhou, M.; Tang, Y.; Deng, X.; Ruan, Ch.; Ding, M.; Shao, J.; Tang, Y.; Wu, Y.

2018. Description of cultivated common buckwheat. In Zhou, M., Kreft, I., Suvorova, G., Tang, Y Woo, S.H. (Eds.). Buckwheat germplasm in the world. Academic Press. Chennai, India. Pp. 53-60. 